\title{
Un Aselle cavernicole d'Espagne méridionale: Proasellus solanasi n.sp. (Crustacea, Isopoda, Asellota)
}

\author{
Jean-Paul HENRY et Guy MAGNIEZ*
}

\section{GÉNERALITÉS}

Les Aselles décrits ici ont été capturés le 20 août 1970, par M.R. Solanas, dans les eaux du système karstique Hundidero-Gato (termino municipal de Benaojan, partido de Ronda, province de Malaga, Espagne méridionale). La Cueva del Gato (Biospeologica No. 623, 6ème série, p. 287) constitue une résurgence du torrent El Gato. Le 9 janvier 1914, elle avait été parcourue par H. Breuil, qui remonta la rivière souterraine sur une centaine de mètres, jusqu'à un petit lac barrant le passage vers l'amont, mais sans découvrir de faune. La Cueva Hundidero (Biospeologica No. 1552, 9ème série, p. 425) est la perte d'un ruisseau épigé et forme une partie amont du réseau hydrographique souterrain. Elle avait été visitée sur quelques centaines de mètres par H. Coiffait, le 7 mai 1952, en période de sécheresse et seule une faune aérienne y fut recueillie.

Les Aselles cavernicoles du système Hundidero-Gato appartiennent au genre Proasellus Dudich que nous avons redéfini récemment (Henry et Magniez 1970). Ils constituent une espèce nouvelle pour la faune ibérique: Proasellus solanasi n.sp., dédiée au prospecteur qui les a découverts. Par ailleurs, nous remercions très sincèrement notre collègue, $M$. Angel Lagar de Barcelone, qui nous a fait parvenir ce matériel, pour la confiance qu'il nous témoigne.

\section{DESCRIPTION SOMMAIRE DE PROASELLUS SOLANASI n.sp.}

Lot capturé: Deux mâles adultes de 7 et $7,4 \mathrm{~mm}$. Bien que les uropodes soient absents, les animaux sont en bon état. Comme par ailleurs ils présentent des caractères morphologiques suffisamment originaux pour qu'aucune confusion ne soit possible, leur description suffit pour donner une diagnose précise de l'espèce nouvelle.

Aspect général: Corps totalement dépigmenté, d'allongement moyen (coefficient 3,3 ), à bords parallèles. Céphalon 1,6 fois plus large que long avec une légère concavité rostrale. Yeux absents. Lobes post-mandibulaires saillants. Largeur maximale du corps au droit du péréionite VI $(2,1 \mathrm{~mm}$ pour le péréionite $\mathrm{VI}, 2 \mathrm{~mm}$ pour le VII et $1,9 \mathrm{~mm}$ pour le pléotelson). Les coxopodites des sept paires de

* Laboratoire de Biologie animale et générale, Faculté des Sciences de la Vie et de l'Environnement 6, bvd Gabriel 21-DIJON 
péréiopodes sont longs et débordent largement les péréionites (ainsi, le péréionite $\mathrm{V}$ mesure $2 \mathrm{~mm}$ en largeur mais 2,2 $\mathrm{mm}$ avec les coxopodites) (Fig. $1 \mathrm{~A}$ ).

Pléotelson subquadrangulaire à peu près aussi long que large, avec pointe caudale peu marquée. Pléonites libres I et II étroits et courts, mais nettement individualisés.

Appendices céphaliques: Antennules de $1,45 \mathrm{~mm}$ plus courtes que la hampe de l'antenne. Hampe de 4 articles et fouet de 8 articles avec 1 lame olfactive de $60 \mu$ sur les 4 articles distaux sauf le dernier.

Antennes de $6 \mathrm{~mm}$; la hampe de 6 articles atteint $1,8 \mathrm{~mm}$ et le fouet de 40 articles $4,2 \mathrm{~mm}$.

Mandibules, maxillules et maxilles typiques du genre Proasellus.

Maxillipèdes: Aspect typique des Asellidae. Endite à 6 crochets rétinaculaires à droite et 7 à gauche. Epipodite bien développé portant 20 petites tiges lisses et marginales à l'angle distal externe et sur le bord externe.

Péréiopodes: Ils sont longs et relativement grêles. Pour l'individu de 7,4 mm, leurs longeurs respectives sont: en mm, 2,49 (I), 3,50 (II), 3,58 (III), 2,84 (IV), 4,71 (V), 5,43 (VI) et 5,71 (VII). Le raccourcissement du péréiopode IV (caractère sexuel o) est donc relativement peu marqué.

Péréiopode I à propodite assez faible. La rangée marginale de phanères ensiformes se poursuit sur la moité distale par une crête garnie de sétules (Fig. I B). Dactylopodites avec 6-7 épines sternales.

La différenciation en crochet nuptial du péréiopode IV existe, mais moins marquée que chez les espèces épigées (Fig. I C). Les dactylopodites des péréiopodes II à VII portent tous 3 épines sternales.

Pléopodes I đ: Protopodite subtrapézoïdal, plus long que large. Bord externe convexe; bord interne très convexe avec un seul crochet rétinaculaire à droite comme à gauche et une courte bordure épineuse plus distale. Exopodite ovalaire, 2 fois plus long que large; il porte 1 ou 2 petites soies lisses sur la marge proximale interne. Une série continue de 7 soies courtes et lisses, subégales, sur le bord externe suivie sur le bord distal de 4 soies lisses plus petites. Présence inhabituelle sur ce pléopode d'une zone pilifère sur la face tergale dans la région distale interne de l'article (Fig. II E).

Pléopodes II o: Protopodite subtrapézoïdal à peine aussi long que large. Près du bord interne, à convexité très accentuée, 4 fortes tiges lisses insérées sur la face sternale de l'article. Exopodite biarticulé très allongé 1 fois $1 / 2$ plus long que le

\section{Planche I -}

A - Moitié droite du péréionite V du ơ de 7,4 mm de Proasellus solanasi n.sp.; e $=200 \mu$.

B - Péréiopode 1 droir du même; $\mathrm{e}=100 \mu$.

C - Péréiopode IV droit du même; $\mathrm{e}=200 \mu$.

D - Pléopode III droit du même; $\mathrm{e}=333 \mu$. 


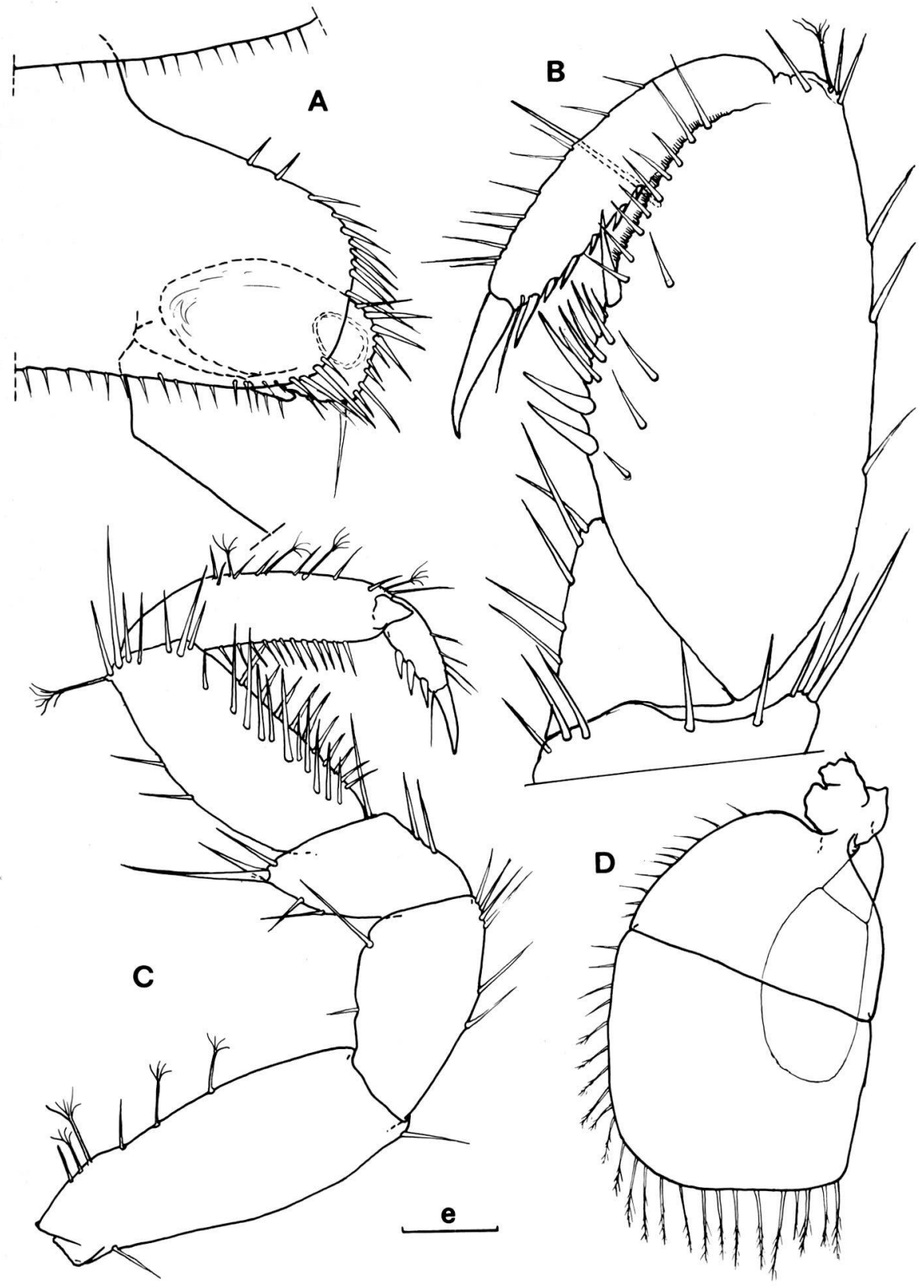


protopodite et l'endopodite. Article proximal court, sensiblement aussi long que large. Article distal subrectangulaire presque 2 fois $1 / 2$ plus long que large et 3 fois $1 / 2$ plus long que l'article proximal. Bords interne et externe rectilignes. Sur le bord distal très convexe, 7 tiges plumeuses d'environ $400 \mu$ de long, c'est-à-dire plus longues que l'exopodite, et 3 tiges plumeuses légèrement plus courtes sur la partie distale du bord externe. Une aire pilifère vers le bord interne-distal de la face tergale (Fig. II F).

Endopodite (organe copulateur) cylindrique, plus large et plus court que l'exopodite. Région proximale à fond légèrement excavé sans apophyse aux angles interne et externe. Région distale typique du genre Proasellus. Le goulot, court et de large diamètre, orienté légèrement vers l'extérieur, et nettement relevé vers la face sternale: son axe détermine un angle de $45^{\circ}$ avec celui de l'endopodite; son extrémité très évasée, avec un rebord irrégulièrement plissé, rappelle l'aspect d'une corolle (Fig. II G). La fente sternale bien visible prolonge celle du goulot. Apophyse tergale conique dirigée vers le côté externe; de plus elle s'incline à $45^{\circ}$ en direction tergale, de sorte qu'elle forme avec le goulot un angle de $90^{\circ}$ environ.

Pléopodes III: Allongement de l'exopodite légèrement inférieur à 1,5. Une suture oblique le divise en 2 articles, le proximal plus petit que le distal. Bord externe de l'article proximal avec 10 tiges courtes et lisses. Article terminal portant une série continue de 25 tiges plumeuses sur les bords externe et distal. Endopodite subovalaire, court et charnu (Fig. I D).

Pléopodes $I V$ : Exopodite 1 fois $1 / 2$ long que large. La linea area délimite une aire respiratoire (area) bien développée. La linea transversalis très oblique divise l'exopodite en 2 articles, le proximal plus court que le distal. La linea conjungens, amorçée seulement au bord externe, marque la limite de la bordure ciliée de la marge externe de l'article proximal. Endopodite charnu bien développé, légèrement moins long que l'exopodite. Ce pléopode est très semblable à celui des Proasellus épigés-oculés à vaste répartition comme $P$. coxalis ou $P$. meridianus (Fig. II. I).

Pléopodes $V$ : Exopodite ellipsoïde environ 1 fois $1 / 2$ plus long que large, divisé en 2 articles par une suture très proximale. Bord externe de l'article proximal garni de 5 soies lisses. Endopodite charnu bien développé moins long que l'exopodite (Fig. II J.).

Planche II -

E - Pléopode I gauche du même, face sternale; l'air pilifère tergale est représentée; $\mathrm{e}=100 \mu$.

$\mathrm{F}$ - Pléopode II droit du même, face sternale; l'air pilifère tergale est représentée; $\mathrm{e}=100 \mu$.

$\mathrm{G}$ - Extrémité de l'endopodite (organe copulateur) du même en vue sternale; $\mathrm{e}=50 \mu$.

$\mathrm{H}$ - Extrémité de l'endopodite du pléopode II ơ de l'espèce Proasellus arnautovici (Remy, 1932) du lac d'Ohrid, d'après Remy, modifié; échelle en $\mu$.

I - Pléopode IV droit, face sternale, du ơ de 7,4 $\mathrm{mm}$ de $P$. solanasi $\mathrm{n} . \mathrm{sp}$.; $\mathrm{e}=200 \mu$.

$\mathrm{J}$ - Pléopode V droit, face sternale du même; $\mathrm{e}=200 \mu$. 
J.P. HENRY ET G. MAGNIEZ

41

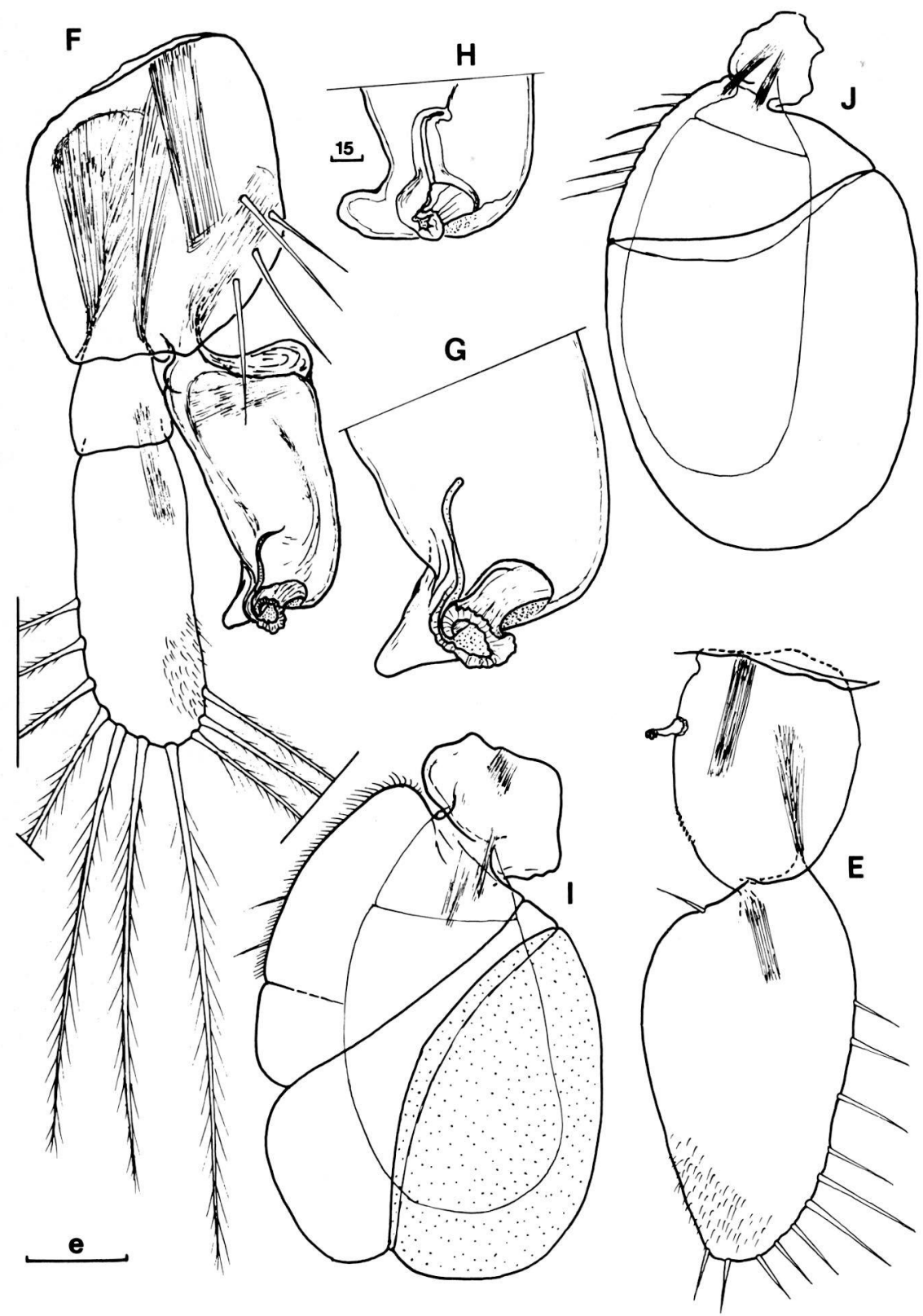


Affinités: Le genre Proasellus compte aujourd'hui une cinquantaine d'espèces déjà décrites, dont la majorité sont des anophtalmes à vie souterraine. Parmi ces anophtalmes, certaines sont des formes de petite taille, gardant à l'état adulte une morphologie juvénile, sans traits originaux permettant de préciser leur parenté immédiate avec les espèces oculées. D'autres au contraire, et c'est le cas de $P$. solanasi n.sp., de taille plus forte, comparable à celle des épigées, laissent plus facilement deviner leurs affinités.

La nouvelle espèce de la province de Malaga montre des affinités indiscutables avec plusieurs espèces pigmentées et oculées du genre. Ce sont d'une part l'espèce à vaste répartition Proasellus meridianus (Racovitza, 1919) de l'Europe occidentale et une forme épigée du Portugal qui en est très proche, P.xavieri (Braga, 1956) et, d'autre part une série d'espèces pigmentées-oculées qui sont considerées comme réprésentant un stock ancien du genre Proasellus; ce sont:

- P. arnautovici (Remy, 1932), du lac d'Ohrid (Yougoslavie), (Fig. II H)

- P. infirmus (Birstein, 1936), d'une source karstique de Transcaucasie occidentale

- P. istrianus (Stammer, 1932), des environs de Trieste.

- P. monodi (Strouhal, 1942), d'une source karstique en Epire.

- P. remyi (Monod, 1932), du lac d'Ohrid.

- Enfin deux formes des eaux karstiques nord-pyrénéennes, considérées précédemment comme proches de l'espèce P. ibericus (Braga) (Magniez 1967, 1970), mais qui en paraissent spécifiquement indépendantes: P. coiffaiti Henry et Magniez; P. racovitzai Henry et Magniez.

Par ses différents caractères, $P$. solanasi n. sp. semble montrer une parenté particulière avec $P$. arnautovici du lac d'Ohrid, notamment en ce qui concerne le pléopode II o (Fig. II G et II H). Des auteurs précédents, Remy (1932) par exemple, ont pressenti que les Asellides périméditerranéens avaient une origine très ancienne. L'expansion des espèces modernes ( $P$. coxalis, $P$. meridianus) ne représente que la dernière phase d'une histoire compliquée. Les espèces oculées endémiques et les anophtalmes qui leur ressemblent, par leur localisation et leur densité, signalent les centres de dispersion originels du genre (Balkans, Péninsule ibérique). Dans cette optique, la découverte de $P$. solanasi est très intéressante. Elle montre un stade morphologique intermédiaire dans la lignée évolutive qui a abouti à Proasellus meridianus en partant des formes endémiques oculées citées plus haut. Mais, comme $P$. solanasi est anophtalme, il ne représente qu'un rameau adjacent de cette évolution.

Manuscrit terminé le 3 juin 1971

\section{RESUME}

Description d'une espèce nouvelle, apigmentée et anophtalme, appartenant au genre Proasellus Dudich. L'Aselle a été capturé dans une rivière souterraine de la région de Malaga (Espagne méridionale). Il appartient à la lignée évolutive menant à Proasellus meridianus (Racovitza). 


\section{SUMMARY}

Description of a new, unpigmented and eyeless species of the genus Proasellus Dudich, from an underground stream in the province of Malaga (Southern Spain). It belongs to the phyletic line which gave rise to the modern epigean water-slater Proasellus meridianus (Racovitza).

\section{BIBLIOGRAPHIE}

BIRSTEIN, J.A., 1936 - Über kaukasische Aselliden. Arch. Mus. Zool. Moskau, 3, 235-242.

BRAGA, J.M., 1946 - Quelques Asellides nouveaux du Portugal. Mem. Est. Mus. Zool. Univ. Coimbra, 173, 9-18.

BRAGA, J.M., 1956 - Un Asellus nouveau de l'étang de Fermentelos (Portugal). Mem. Est. Mus. Zool. Univ. Coimbra, 245, 1-12.

HENRY, J.P., 1971 - Données récentes sur la faune hypogée d'Asellides de France. Actes $96^{\circ}$ Cong. Nat. Soc. sav., Toulouse, sous presse.

HENRY, J.P. et MAGNIEZ, G., 1970 - Contribution à la systématique des Asellides. Ann. Spéléol., Paris, 25, 335-367.

MAGNIEZ, G., 1970 - Sur un Aselle cavernicole de France: Proasellus ibericus (Braga). Sous le Plancher Dijon, 9,21, 25.

MARGALEFF, R., 1953 - Algunos crustaceos de las aguas continentales ibericas. Inst. Florestal Invest. exp. Minist. Agric. Madrid, 171-185.

MONOD, Th., 1932 - Sur un Asellus aberrant (A. remyi n.sp.) du Lac d'Ohrid. Bull. Soc. Zool. France, 57, 200-217.

RACOVITZA E.G., 1919 - Notes sur les Isopodes. 2. Asellus aquaticus L. et A. meridianus n. sp. Arch. Zool. exp. gén., Paris, 58, 31-43.

REMY, P. - Description d'Asellus (Proasellus) arnautovici n. sp. du lac d'Ohrid. Bull. Soc. Hist. nat. Moselle, 33, 73-87.

STAMMER, H.J., 1932 - Zur Kenntnis der Verbreitung und Systematik der Gattung Asellus, insbesondere der mitteleuropäischen Arten (Isopoda) Zool. Anz., 99, 113-131.

STROUHAL, H., 1954 - Zoologische Studien in West-Griechenland: Süsswasser-Isopoden. Österr. Ak. math. naturw. Kl. 163, 11-44. 